\title{
Photometric properties of facular features over the activity cycle
}

\author{
I. Ermolli ${ }^{1}$, S. Criscuoli ${ }^{2,3}$, M. Centrone ${ }^{1}$, F. Giorgi $^{1}$, and V. Penza ${ }^{2}$ \\ 1 INAF - Osservatorio astronomico di Roma, via Frascati 33, 00040 Monte Porzio Catone, Italia \\ e-mail: ermolli@oaroma.inaf.it \\ 2 Dipartimento di Fisica, Università di Roma “Tor Vergata", via della Ricerca Scientifica 1, 00133 Roma, Italia \\ 3 High Altitude Observatory, 80307-3000 Boulder, CO, USA
}

Received 7 July 2006 / Accepted 18 November 2006

\begin{abstract}
Aims. We have analyzed the contrast of facular features identified in a large dataset of PSPT full-disk photometric images and SoHO/MDI magnetograms, obtained from 1998 to 2005. The aim of this work is to contribute to the improvement of semi-empirical atmospheric models and of irradiance studies and to understand the reasons for the controversial results of facular contrast already presented in the literature.

Methods. We used different identification methods to analyze their effects upon the results obtained. We also analyzed the effects of the limited information content in the analyzed images.

Results. We show that selection effects associated with the identification method may produce significant differences in the results. The facular contrast is not only a function of both selection methods and the heliocentric angle, but also of feature size, activity level, and content of the analyzed images. Comparisons of the results obtained with computations of the most recent semi-empirical atmospheric models of facular features show that these models reproduce limb-angle corrected contrast measurements with an offset up to $\approx 1 \%$ from the disk center to $\mu=0.3$.
\end{abstract}

Key words. Sun: activity - Sun: photosphere - Sun: chromosphere - Sun: faculae, plages - techniques: images processing

\section{Introduction}

The space-based measurements of solar irradiance variations obtained since 1978 have been recently reproduced by using one-dimensional time-independent semi-empirical atmospheric models of solar features and observed properties of the modelled features, specifically the fraction of solar surface covered by a given feature at any given time (Krivova et al. 2003; Penza et al. 2003; Fontenla et al. 2004; Wenzler et al. 2005).

These reconstructions use complementary approaches and are different in a few aspects, e.g. the number and the temperature structures of the atmospheric models adopted in order to compute the brightness of the observed features, the spectral synthesis program employed, as well as the data used to identify features of the quiet and active sun. Notice that the comparison between reconstructed and measured irradiance variations show that more than $90 \%$ of the measured variance is explained through these models (Solanki \& Krivova 2004). However, both the unexplained variance and some shortcomings of the models presented show that further work is required. In particular, semiempirical models describing bright regions should be improved (Fontenla et al. 2004; Penza et al. 2004).

Facular regions are the photospheric, small bright patterns around dark sunspots and in the remnants of active regions, originally discovered near the limb in full-disk white light images. Co-spatial chromospheric regions observed in the CaII K spectral range are bright as well. The connection of facular regions to strong magnetic field was stated by Chapman \& Sheeley (1968) and Frazier (1971), while their sub-arcsecond resolution imaging was obtained by Dunn \& Zirker (1973) and Mehltretter (1974). Starting from earlier photometric studies (Richardson 1933), facular contrast ${ }^{1}$ has been reported by negative, zero, or positive values near the disk center. There are controversial results for the extreme limb, too: here sometimes the contrast appears to fall off, while other times it appears to increase sharply (for a review of earlier results, see e.g. Ahern \& Chapman 2000; Walton et al. 2003; Steiner 2005). Differences in the reported measurements have been ascribed to feature-discrimination method effects, i.e. the facular contrast being not only a function of the heliocentric angle, but also of wavelength range, facular size, magnetic field strength, spatial resolution, activity level, and selection effects (Solanki 1993, 1994).

Recent high-resolution images show facular brightening in unprecedented detail (Lites et al. 2004). These appear as relatively extended features about 0.6 arcsec on the centerward side of solar granules and show a high temporal variability. Three-D numerical MHD simulations of a non-grey atmosphere (Keller et al. 2004) reproduce the observed small-scale features. The authors of these simulations give an explanation of facular brightening basically in accordance with the "flux-tube bright wall" model of Spruit (1977).

We present the results obtained by analyzing the center-tolimb variation (CLV) of facular contrast by using a large dataset of full-disk photometric images and magnetograms. We studied the properties of features identified through different methods, some of which have already been presented in the literature. We also analyzed the dependence of the measured CLV on several observation constraints, such as spectral range, spatial resolution, and activity level. The objective of this study is

\footnotetext{
1 The contrast is defined as $\frac{I_{\mathrm{f}}-I_{\mathrm{q}}}{I_{\mathrm{q}}}$, where $I_{\mathrm{f}}$ is the intensity of the facular region and $I_{\mathrm{q}}$ the intensity of the quiet sun at any equivalent position on the solar disk.
} 
twofold. First, it contributes to improving atmospheric models assumptions, widely used for both irradiance studies and numerical simulations of the solar atmosphere. Second, it singles out an identification method based on CaII K observations, like those available for reconstructions of past total solar irradiance variations. This method reproduces the outcome of current measurements based on magnetic signals. Moreover, this analysis makes us understand the differences in results presented in the literature.

The paper is organized as follows. In Sect. 2 we present the data sets used and the pre-processing applied to the images analyzed. In Sect. 3 we describe the five methods utilized to identify facular regions. In Sect. 4 we show the results obtained, which are discussed in Sect. 5. Finally, Sect. 6 presents our conclusions.

\section{Observations and image pre-processing}

\subsection{Data sets}

The bulk of the data we analyzed derives from the archive of the daily full-disk observations carried out with the PSPT telescope at the Rome Observatory. This archive ${ }^{2}$ contains images taken since July 1996. However, the data were obtained with the final version of the telescope only from October 1997 onwards. The PSPT was designed (Coulter \& Kuhn 1994) to provide highprecision photometric full-disk observations, and the current instrument operation is described by Ermolli et al. (2003). Briefly, images are acquired with a $2048 \times 204812$ bit pixel CCD camera, $2 \times$ binned, with a final spatial scale of $\approx 2$ arcsec pixel, using three interference filters centered on the CaII K line $(393.3 \mathrm{~nm}$, fwhm $0.25 \mathrm{~nm})$, blue continuum $(409.2 \mathrm{~nm}$, fwhm $0.25 \mathrm{~nm})$, and red continuum $(607.1 \mathrm{~nm}$, fwhm $0.5 \mathrm{~nm})$.

From the full archive we selected daily "sets" of CaII K, blue and red images, obtained on 291 different observing days, during the months between July and September, from 1998 up to 2005, i.e. under the same instrumental and operational setups. We chose to analyze images obtained during these months, because these were taken with better observing conditions (weather, short exposure times, solar declination). Each "set" of CaII K and continuum images analyzed were taken within a few minutes (less than $5 \mathrm{~min}$ ) from each other, so as to achieve nearly simultaneous observations of the solar photosphere and chromosphere.

The largest sample of the images analyzed was obtained by co-adding frames acquired with very short exposure times (usually less than $50 \mathrm{~ms}$ ). The addition of frames is meant to decrease the noise of the photometric measurements, which is on average lower than $\approx 0.1 \%$ per pixel after instrumental calibrations. In general, the addition of frames decreases the spatial resolution on images to about 3 arcsec (Fazzari et al. 2003). Thus, singleframe images acquired in the year 2005 were analyzed as well, to evaluate degradation effects induced either by seeing changes or by instrumental characteristics.

We also analyzed a sample of 42 images acquired from July to September 2005 with the PSPT telescope operated by the High Altitude Observatory at the Mauna Loa Observatory (MLSO). These images were acquired with a "twin" telescope ${ }^{3}$, at wavelengths similar to those mentioned above.

The MLSO PSPT archive contains observations taken since 1998. Nevertheless, in 2004 some major upgrades were installed, affecting both telescope operation and characteristics. Due to both telescope operation and site, MLSO images are characterized on average by a better spatial resolution and a lower

\footnotetext{
2 http://www.mporzio.astro.it/solare/

3 http://rise.hao.ucar.edu/
}

scattered light-level, as compared to the Rome images. For instance, the pixel scale is 1 arcsec in the MLSO images, instead of 1.9 arcsec in the Rome ones. For each observational day, the images of best quality obtained at MLSO were selected (details are given by Criscuoli et al. 2007).

Finally, we analyzed MDI full-disk magnetograms (Scherrer et al. 1995), selected from those available in the SoHO archive ${ }^{4}$ during the months between July and September from 1999 to 2005. There are no MDI observations for the same months in 1998, due to SoHO spacecraft problems. Among all the data available, we had to search for the full-disk magnetograms acquired almost simultaneously to the Rome PSPT images. The analyzed data set consists of 237 magnetograms, recorded during 112 days spread over the period described above. We tried to maximize the number of usable MDI data, but we found difficulties in obtaining magnetograms close in time to the PSPT observations. In all the analyzed images, MDI and PSPT data were recorded within $10 \mathrm{~min}$ from each other.

The MDI data provide a measure of the line-of-sight component of the magnetic field averaged over a $2 \times 2$ arcsec resolution element. We mainly used single magnetograms acquired with $30 \mathrm{~s}$ integration time. Nevertheless, we have also analyzed two samples of magnetograms acquired during the year 2000, with $30 \mathrm{~s}$ and $300 \mathrm{~s}$ integration times, as well as averages of single magnetograms, so as to evaluate how much the results are affected by the reduction of the data-noise level.

\subsection{Image pre-processing}

The images from the Rome archive were pre-processed in order to apply the instrumental calibration, i.e. dark and flat-field compensations, as described in Ermolli et al. (2003). The centerto-limb variation of the quiet sun was then compensated for by using the average radial profile of the intensity over the solar disk. Active regions were excluded from this computation by an intensity threshold criterion.

The MLSO images we used were independently dark and flat-field corrected. They also had the mean center-to-limb variation independently removed. The procedures applied to these data are different from those used with the Rome images and are described by Meisner \& Rast (2002).

All the PSPT and MDI images were first re-sized to the same solar disk size, by assuming linear interpolation. Then images were rotated and co-aligned in order to allow a comparison pixel by pixel (Fig. 1).

Following the authors who analyzed MDI full-disk magnetograms (e.g. Ortiz et al. 2002; Wenzler et al. 2004; Domingo et al. 2005; Ortiz et al. 2006), we determined the data-noise level as a function of solar disk position. This is done by computing the standard deviation (sdev) for the magnetic signal with a running box (box side $=40$ pixel) on each magnetogram of a data sample acquired in a low activity period. The magnetograms analyzed for the year 2005 were used to this purpose. In order to reduce effects of a possible remaining activity, the median pattern was then evaluated from the set of computed sdev images. Moreover, a further smoothing filter is applied to the median pattern, so as to get the large-scale variation of the data noise. The pattern obtained was used to compensate for the observed increase toward the limb of the noise level on each analyzed magnetogram. Notice that, due to the running box side, the solar disk we analyzed is reduced by about $8 \%$. A steady

\footnotetext{
4 http://soi.stanford.edu/data/
} 


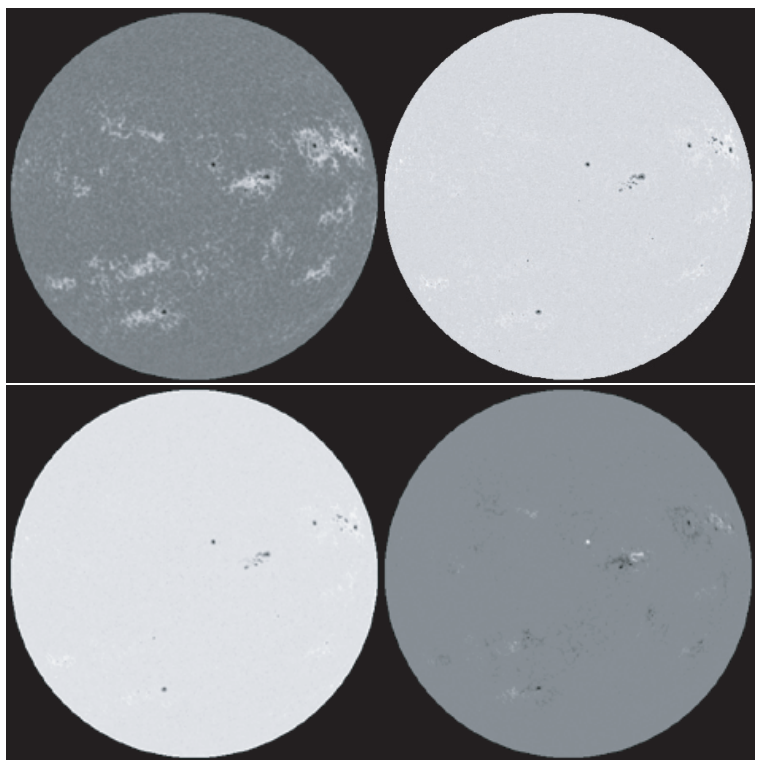

Fig. 1. Examples of the full-disk images analyzed for this study, obtained on July 26, 2000. Top: Rome PSPT CaII K (left) and blue continuum (right) images. Bottom: Rome PSPT red continuum image (left) and MDI full-disk magnetogram (right).

extrapolation of the evaluated pattern is then computed in order to cover the whole solar disk.

\section{Facular region identification}

Facular regions were identified on the pre-processed images by applying five automated methods (Fig. 2).

Method 1: we applied the method presented by Nesme-Ribes et al. (1996). It (NR, hereafter) corresponds to a straight thresholding of contrast images. The threshold value is obtained for each image $i$ by studying the variation in the mean quiet sun intensity for different thresholds. The threshold value that minimizes the quiet sun intensity is assumed as the reference threshold $K_{i}$. It is worth noticing that the "reference threshold value" $K_{i}$ is relative to a single image $i$ and partly depends on the image quality. Moreover, it slowly varies in anti-phase with the solar activity, most likely because of unresolved small-scale magnetic elements. Since this can affect studies of the dependence of facular contrast on solar activity, we used a single "average reference threshold value" $K$ for all our datasets for the time-variation analyses presented in Sect. 4.3. The value we chose is the average of the "reference threshold values" $K_{i}$, as computed for images from the year 2000 .

Method 2: we applied the method presented by Ahern \& Chapman (2000). It $(\mathrm{CH})$ is based on a contrast threshold relative to the quiet sun that varies as $I_{\mathrm{f}}=$ threshold $\times I_{\mathrm{q}} / \mu$, where $I_{\mathrm{f}}$ is the intensity of the facular region, $I_{\mathrm{q}}$ the intensity of the quiet sun, $\mu=\cos \theta$, and $\theta$ is the heliocentric angle. We assumed the threshold as the "average reference threshold value" $K$ resulting from the $N R$ method described above.

These two first methods take only the brightness properties of pixels into account to single out features. Since intensity distributions of network and facular regions usually overlap, methods that are based only on intensity criteria may fail to single out these two classes of features.

Method 3: we used a criterion based on intensity, continuity, and size of features $(K t r)$. We first extracted relevant regions of the solar disk that include active regions and their remnants (see

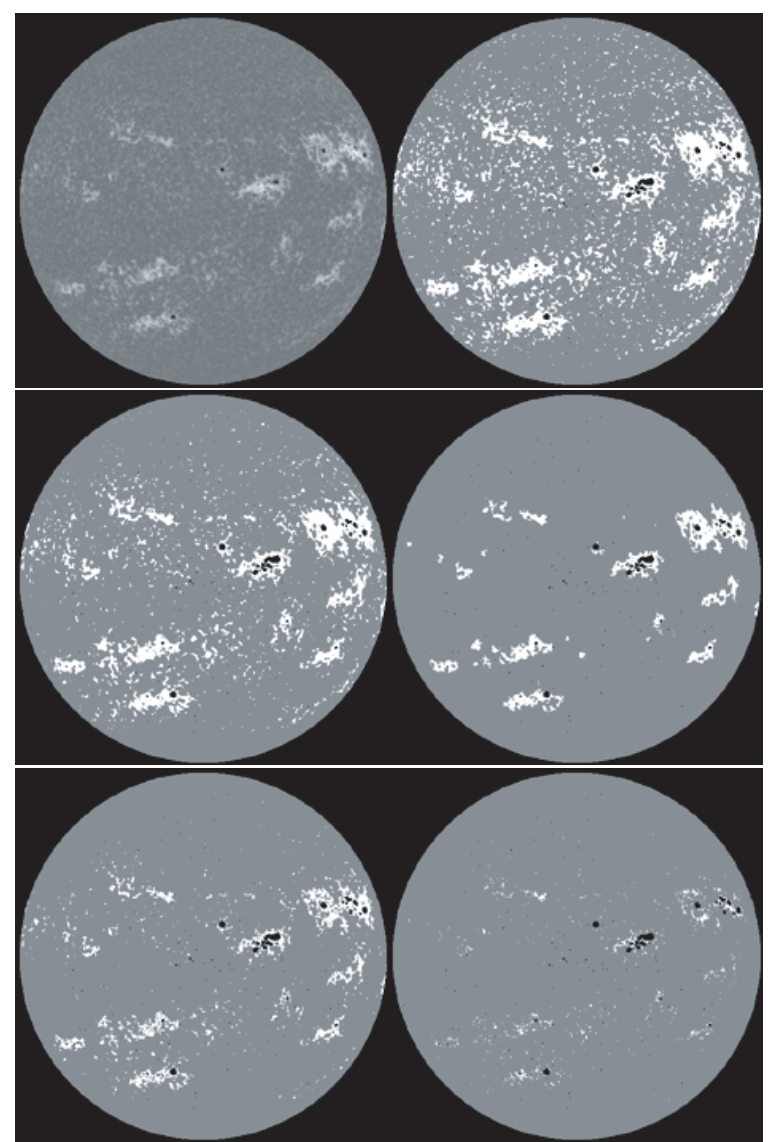

Fig. 2. Examples of the results obtained by the processing of the images shown in Fig. 1. From the left, top: Rome PSPT CaII K image and binary image obtained by the $N R$ method; middle: binary images obtained by the $C H$ and Ktr methods; bottom: binary images obtained by the the $B-R$ and the $M a g$ methods.

e.g. Fig. 1 of Caccin et al. 1998). These pre-selected regions are made up of all those pixels that are both brighter than a fixed contrast value and larger than a fixed size, on a spatially filtered image (box side $=\frac{R_{\text {sun }}}{20}$ ). The "reference threshold value" $K_{i}$ obtained with the $N R$ method is then used to single out from the pre-selected regions all those features identified for our study.

Method 4: this method uses the photometric properties of facular features in the photosphere $(B-R)$. In particular, each day we compute a color image by subtracting the two continuum images (blue minus red), in order to isolate the facular regions by differential photometry, as proposed by Foukal \& Duvall (1985). We identified the photospheric facular regions by thresholding the color image, with the threshold value set to $\sigma$ of the color image intensity.

Method 5: we used a threshold to select facular features by their signature on magnetograms $(\mathrm{Mag})$. Following the authors who analyzed MDI data with this criterion (e.g. Wenzler et al. 2004; Ortiz et al. 2006), we set the threshold value to $\pm 3 \sigma$ of the magnetogram values. Actually, we used data values weighted by the factor $\frac{1}{\mu}$, so as to take into account that the magnetograms underestimate the magnetic flux in pixels close to the limb. Moreover, we applied a further $3 \times 3$ smoothing filter to remove isolated pixels. These pixels are identified mainly near the limb, because the noise is considerably larger there than at the disk center.

Through the different methods applied, we obtained five binary images with masks of the identified facular regions for each 

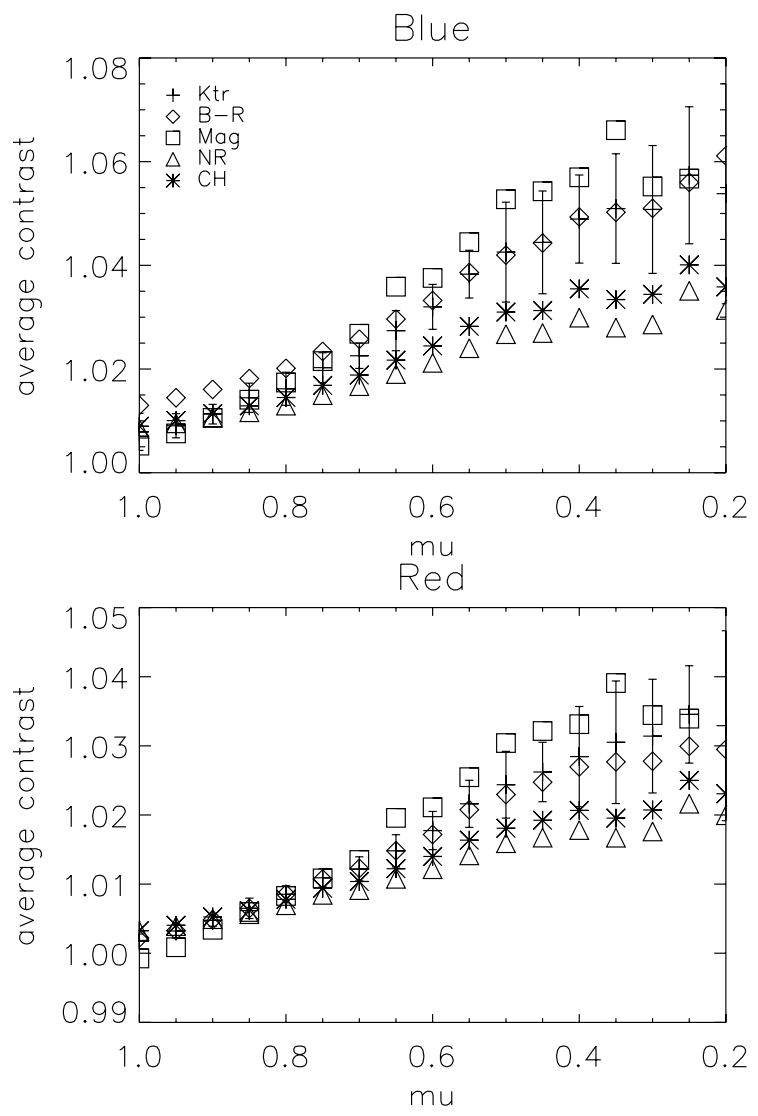

Fig. 3. Center-to-limb variation CLV of the facular contrast in the two PSPT continuum bands (top = blue, bottom $=$ red measurements) computed for the year 2000, using the five methods described in Sect. 3. Error bars represent the standard deviation over the position bin; for clarity, they have been superimposed only over the results obtained with the Ktr method. Details about the deviation of contrast results obtained with the other methods are given in the text.

day. We exclude from these masks all the pixels that are identified on the corresponding PSPT red continuum images by their contrast, below the $3 \sigma$ average contrast. In this way, possible isolated dark pixels are also removed from the identified features.

In brief, the different methods applied use the datasets analyzed as follows: to identify facular features $\mathrm{NR}, \mathrm{CH}$, and $\mathrm{Ktr}$ use the PSPT CaII K images, $B-R$ uses the PSPT red and blue images, and Mag the MDI magnetograms. In order to exclude spots and pores, all methods use the PSPT Red images. The contrast of the identified regions is defined, for each heliocentric angle, as the ratio between the mean intensity of the facular pixels belonging to the angle and the mean value of the quiet sun intensity. Notice that a definition of contrast based on median values may slightly modify $(<0.5 \%)$ the results, without affecting the general discussion presented in the following.

Identified facular regions are also labelled to perform the study of the dependence of contrast on size, as described in Sect. 4.2.

\section{Results}

\subsection{Contrast CLV}

The five methods single out chromospheric regions that are characterized by different average contrast values. The relative difference between maximum and minimum values obtained, which are achieved with Mag and NR, respectively, is about $15 \%$ in the
PSPT CaII K band. The contrast values of CaII $\mathrm{K}$ features identified by $N R, C H$, and $K t r$, as well as the deviation in measured values, do not show a disk position dependence. The contrast values are $\approx 1.24,1.24,1.34$, for the three methods. The deviation of measured values is roughly the same for all the three methods, specifically \pm 0.02 . By contrast, the results obtained with $B-R$ and Mag show a monotonic increase in the average contrast of features from the disk center to $\mu=0.2$. This increase is about $1 \%$ and $5 \%$, for the two methods. The standard deviation in measured values also slightly increases toward the limb. The mean contrast at the disk center is $\approx 1.31 \pm 0.02$ and $1.35 \pm 0.04$ in the two methods respectively.

Figure 3 shows the average contrast CLV in the PSPT blue and red bands. In all cases, we find that the contrast CLV increases almost monotonically toward the limb, with no maximum up to $\mu=0.2$. Actually, the CLV obtained with Mag shows nearly a plateau for disk position corresponding to $\mu<0.4$, but this is likely to be due to the applied compensation of the noise pattern.

Notice that the contrast CLV obtained depends largely on the identification method used. In particular, the results differ for up to a factor $3 \%$ and $2 \%$ at $\mu=0.2$ on PSPT blue and red bands, respectively.

We find that the contrast of features selected with Ktr is slightly larger than that computed by all the other methods, save the one of the magnetic signal. Actually, Ktr selects larger size features that mainly occur on activity belts. Mag identifies much smaller size features than do the other methods, features that are also not strictly distributed along activity belts, but mostly occur there. We find that the average contrast of these features is slightly larger than the one obtained by $\operatorname{Ktr}(>1 \%$ at $\mu<0.5)$. On the other hand, the contrast values obtained for the features identified at the disk center by Mag is lower than the values measured with the other selection criteria $(\approx 0.5 \%)$. Average contrast values lower than unity, i.e. features that show a negative contrast relative to the quiet sun, are found strictly at the disk center of the red band observations. Negative contrast measurements are found up to $\mu=0.9$, but the average value is positive.

The vertical bars in Fig. 3 show the standard deviation of measured values. At each disk position, this deviation also depends slightly on the applied identification method. In particular, dispersion of contrast values obtained by $K t r$ and $B-R$ are very similar. The one obtained by $C H$ and $N R$ is smaller, up to half the value at disk positions $\mu>0.6$. On the other hand, the deviation of values obtained by Mag is up to 1.5-2 larger than the one obtained by the other methods at each disk position.

The maximum contrast and its deviation vary in a similar fashion to the average values, though its dependence on disk position is twice as large. The average value of the maximum contrast obtained at each disk position does not depend on the method. In fact, the absolute difference among the obtained values is smaller than the deviation of the contrast measurements at each disk position. On the contrary, the average of the minimum contrast value depends slightly on the method applied for disk position $\mu<0.8$. Nevertheless, the absolute difference among the obtained values lies in the largest deviation computed. The average of the minimum value obtained is negative at each disk position for both the continuum bands analyzed.

As discussed below, the increased deviation in the data towards the limb shown in Fig. 3 is likely due both to increased spatial smearing in the smaller facular regions there and to real contrast differences between individual features.

In Fig. 4, we show the ratio of the average contrast value measured in the two continuum bands versus disk position. As 


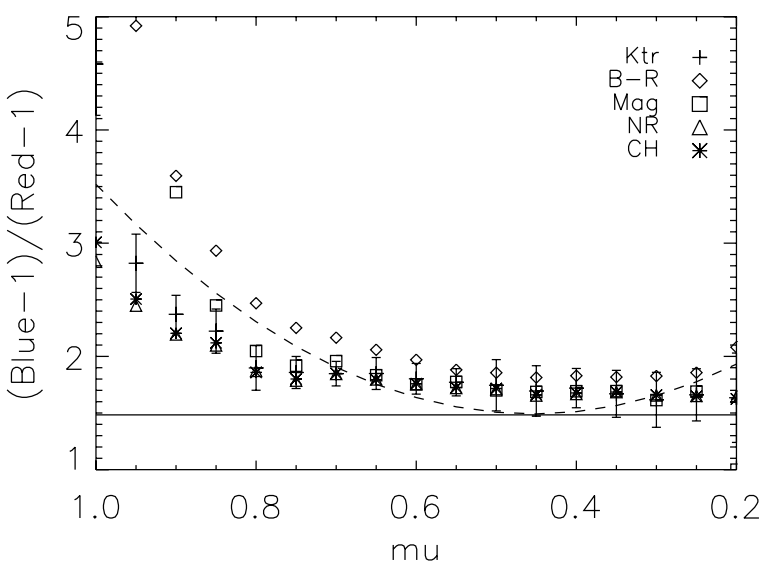

Fig. 4. Ratio of the contrast CLV dependences measured by the different identification methods. For clarity, the deviation of ratio values has only been superimposed over the results obtained with the Ktr method. The dashed line represents a 2nd-order fit to these measurements, while the solid line shows the fit obtained with a black-body approximation.

reported by Allen (2000), the wavelength dependence of facular contrast is approximately given by $C_{\lambda}(\mu)-1=\left(C_{5300}(\mu)-\right.$ 1) $0.5 \lambda^{-1}$ where $C_{5300}(\mu)$ is the intensity of the facular features relative to the photosphere at $5300 \AA$. We find that the ratio of measured CLV dependences is also sensitive to the identification method applied, in particular near the disk center. Moreover, we find that the linear relation drawn by the approximation lies systematically below the curves obtained from all the measured values.

\subsection{Size dependence}

Figure 5 shows the variation in the contrast of features as a function of their size. This variation concerns features identified at the disk center $(\mu>0.9)$ with $K t r$ on the entire dataset of Rome PSPT images. We investigated the scaling of the maximum and average contrasts of the features with their area, the latter being defined by the number of pixels selected by each identified feature. Contrast values were averaged in area bins of width $\Delta \log A=0.05$ (see detail in Criscuoli et al. 2007). In order to reduce projection effects, the analysis was restricted to features whose geometrical barycenter occurs in disk-position bins of width $\mu=0.1$.

We find that the average contrast of facular features at the PSPT CaII $\mathrm{K}$ band increases with feature size for area values lower than about $2000 \mathrm{pixel}^{2}$. This area value corresponds to about $2800 \mathrm{Mm}^{2}$. The contrast oscillates around the mean value $1.30 \pm 0.02$ at area values higher than $2000 \mathrm{pixel}^{2}$. On the other hand, the average contrast of corresponding photospheric features on the PSPT continuum bands seems roughly constant, with a slight decrease in the measured values for large features. For clarity, the photospheric values have been multiplied by a factor 20. Finally, the maximum contrast value on the identified features increases with the feature's size for all the three sets of analyzed images.

We only show the result obtained by the features identified at the disk center with Ktr. Nevertheless, this result is observed also for the features identified with the other methods applied, as well as considering other disk positions. Notice that the number of features identified at the disk center $(\mu>0.9)$ by $N R, C H$, and $B-R$ is about 10 times larger than the one obtained by Ktr, and 3 times larger than the one obtained by Mag.
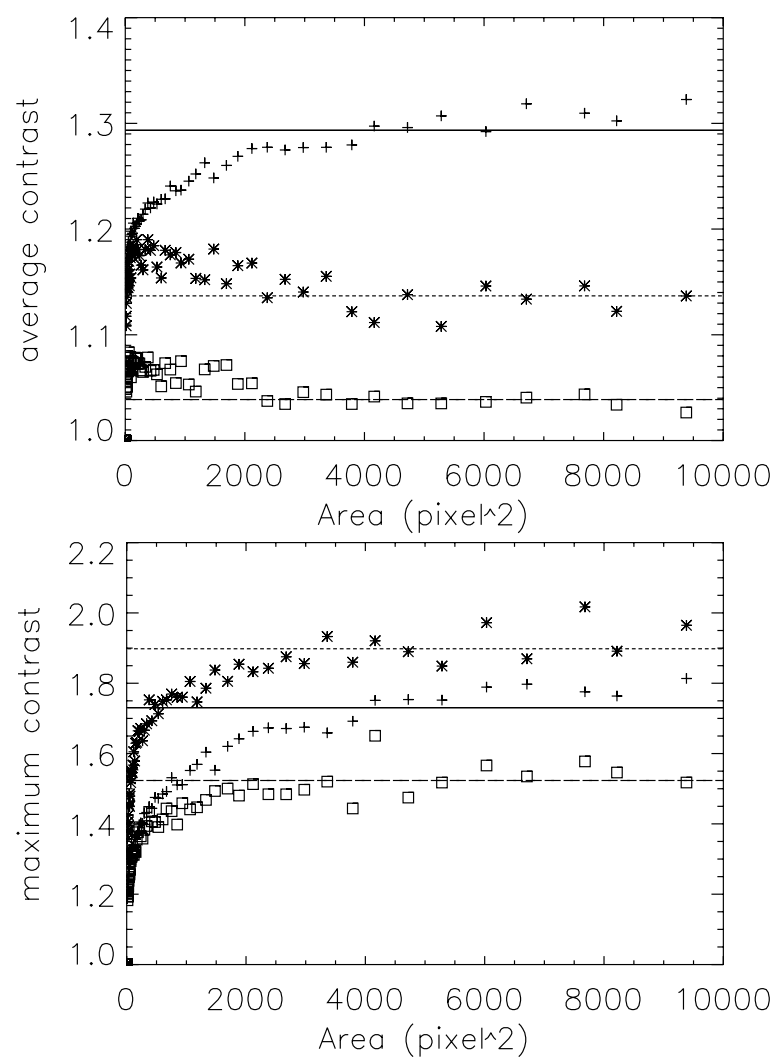

Fig. 5. Dependence of average (top) and maximum (bottom) contrasts of features identified by the Ktr method in their area (crosses, stars, and square show CaII K, blue and red bands results, respectively). For clarity, the continuum contrast dependences have been scaled $(20 \times)$. The horizontal lines represent the mean contrast values for features with an area larger than 2000 pixel $^{2}$ (solid, dotted, dashed lines show CaII K, blue, and red measurement results, respectively).

Trends similar to the one shown in Fig. 5 were also observed when analyzing features identified in subsets of images acquired during the same year.

\subsection{Time variation}

Figure 6 shows the relative differences between the contrast CLV of different years and the one obtained for the year 2000. The features analyzed were identified by Ktr. It appears that the contrast CLV does not change significantly with time. In particular, the relative differences among the contrast CLV for the different years are smaller than $1 \%$ and $0.5 \%$ for the blue and red continuum bands, respectively, up to $\mu=0.5$. These differences increase toward the limb. Here they also seem to follow the specific temporal order of the decrease in activity cycle 23 , mainly those from the PSPT red measurements.

We suggest that this apparent cyclic variation may be due to sample effects. In fact, an increase in the observed relative differences may arise from the decreased number of facular regions identified on the disk at the decrease in solar activity. The limited samples of features identified toward the limb for the years 1998, 1999 and 2004, 2005 are probably the main source of deviation in this measurement.

This interpretation is confirmed by the time dependence of the contrast of features separated by their size, i.e. taking separately into account those features whose area is larger or smaller than a reference area value. As shown in Fig. 7, both the average 

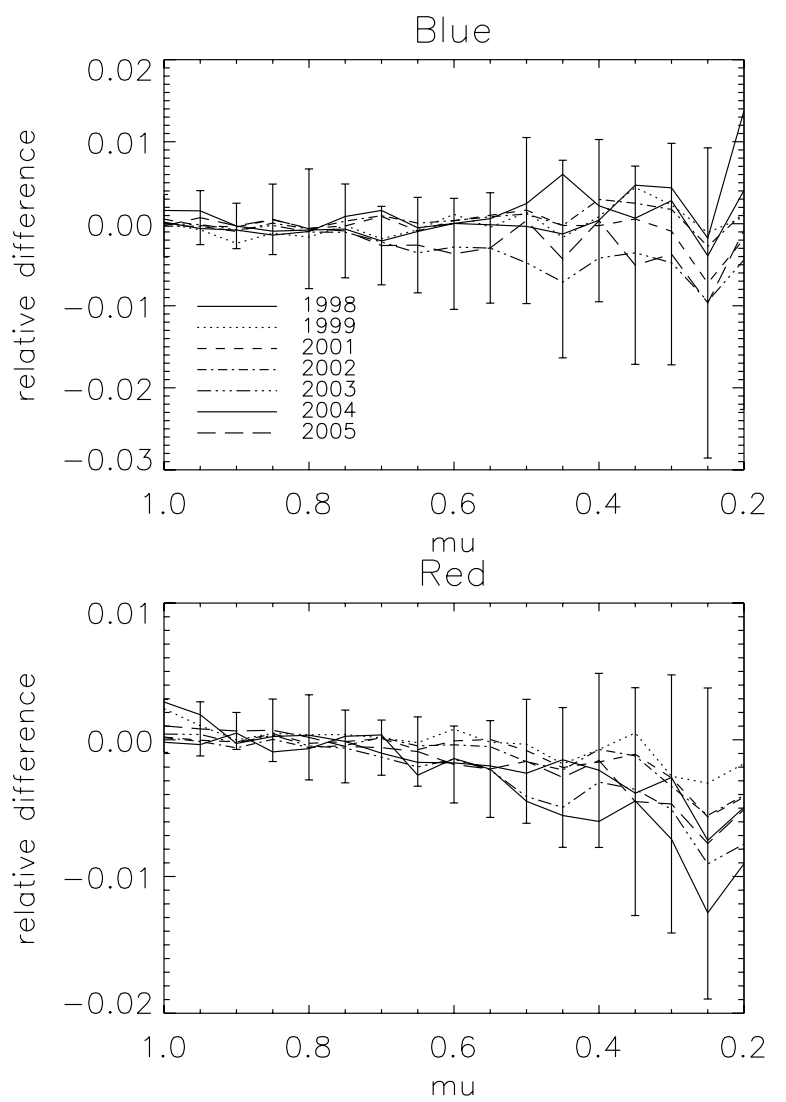

Fig. 6. Relative differences in yearly facular contrast with respect to the one obtained for the year 2000 as a function of the disk position (top = PSPT blue, bottom = PSPT red measurements). Each curve represents the relative difference, for each year, between the calculated contrast CLV and the one obtained for the year 2000. For the sake of clarity, deviation is plotted only for the curve with the highest difference values.

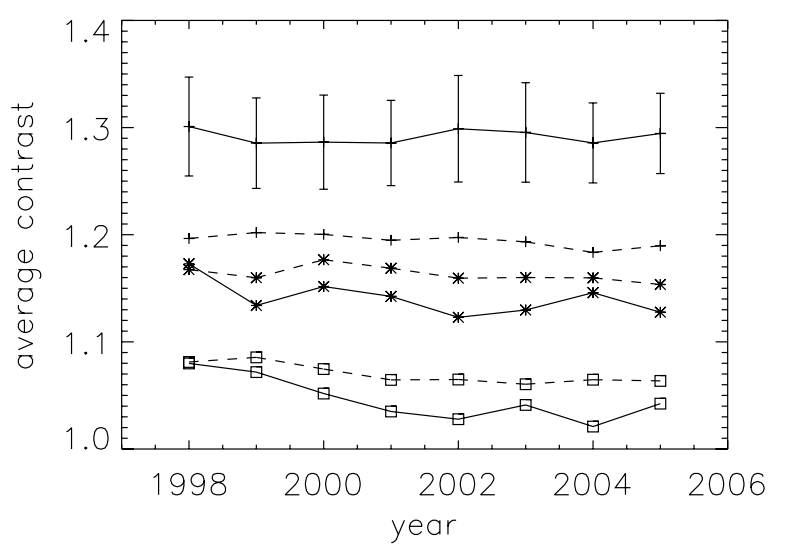

Fig. 7. Temporal variation in the average contrast of features identified at disk center $(\mu>0.9)$ and selected depending on their size: features with area larger (solid line) and smaller (dashed line) than 2000 pixel $^{2}\left(\approx 2800 \mathrm{Mm}^{2}\right)$. Crosses, stars, and squares show the CaII K, blue, and red measurement results, respectively. For clarity, the deviation in measurements is plotted only for the sample with the highest contrast values.

and the maximum contrasts of features identified at disk center $(\mu>0.9)$ and separated by their size for each year of the period analyzed show variations within the deviation in measurements. The reference area value considered is $2000 \mathrm{pixel}^{2}$. The choice of this area value lies in the contrast dependence on size shown in Fig. 5.

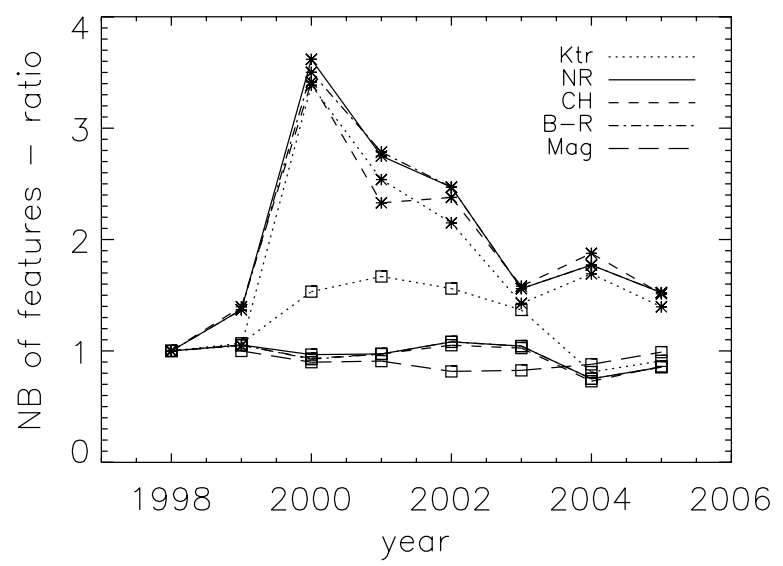

Fig. 8. Temporal variation in the number of features identified at disk center $(\mu>0.9)$ by the five identification methods applied. Symbols refer to features with area smaller (squares) and larger (stars) than $2000 \mathrm{pixel}^{2}$. The choice of this area value is based on the contrast's dependence on the size shown in Fig. 5. The number of identified features is normalized as described in the text.

We also find that the size distribution of the features identified evolves through the period analyzed following the solar cycle phase. For instance, Fig. 8 shows the temporal changes of the number of features identified at the disk center by the five methods applied, with an area larger or smaller than 2000 pixel $^{2}$. The numbers of features identified by each method have been normalized to the number of images analyzed in the year and then to the number of corresponding features obtained for the year 1998. Mag results have been normalized to the number of features obtained for the year 1999.

We find that small-size features identified by $\mathrm{NR}, \mathrm{CH}, \mathrm{B}-\mathrm{R}$, and Mag are almost equally present throughout the period analyzed. Small-size features selected by Ktr vary with the activity level, with a maximum over minimum ratio $\approx 1.5$. The number of larger-size features identified by $K t r, N R, C H$ and $B-R$ shows a similar cycle variation, with a maximum over minimum ratio $\approx 3.5$. The number of small features always outnumbers the larger ones, with a ratio factor of $\approx 200$ and 20 for $N R$ and $K t r$, respectively.

It is worth noticing that the results presented are obtained by assuming a fixed intensity threshold value, to identify features over the whole sample of images. In detail, we assumed the "average reference threshold value" $K$ obtained for the year 2000 . Larger contrast CLV variations that are well correlated with activity level were obtained when applying methods in which threshold values are determined by analyzing of the intensity distribution content of images. Nevertheless, these CLV variations are mostly due to the variation in the intensity distribution of the analyzed images, which, as we have shown, vary with the solar cycle.

\section{Discussion}

This study evaluates the dependence of facular photometric properties on the method applied to identify regions, as well as on feature size and the activity level.

We notice that the different identification methods applied correspond to five operative definitions of the analyzed features. These definitions differ from those used in other works (e.g. Harvey \& White 1999; Turmon et al. 2002; Walton et al. 2003). There is no universally-accepted, unique nomenclature for solarsurface features. With respect to the scheme of feature classes 


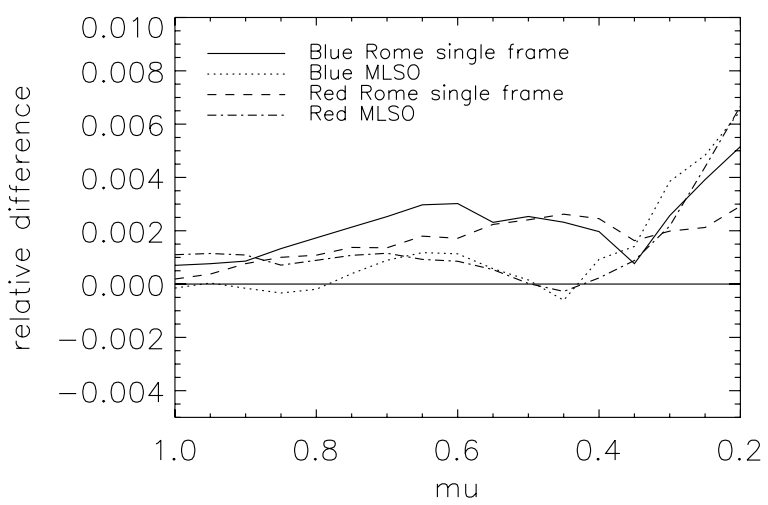

Fig. 9. Relative difference among the contrast CLV dependences obtained by analyzing higher resolution MLSO and Rome PSPT images, compared to those used for the measurements reported above.

developed by Harvey \& White (1999), regions identified by our methods include plages, active and decaying active regions, as well as enhanced network elements. We label all these features as facular regions.

We notice that some of the results presented, especially those concerning the quantification of contrast dependence on feature size, area coverage, and variation in time, may depend on details of the identification criteria used. For instance, an increase in the threshold value used by Ktr modifies the contrast CLV obtained with that method. The absolute differences between the contrast values obtained with the two thresholds are slightly larger than the deviation in measurements at disk positions $\mu<0.5$.

Finally, we notice that the scaling of facular size with atmosphere height (Chapman \& Sheeley 1968) is unlikely to be resolved by the data analyzed in this study, due to pixel size and spatial resolution. We thus consider co-spatial photospheric and chromospheric features.

\subsection{Observational and computational limitations}

The results presented above were obtained from analyzing a sample of best-quality images extracted from the Rome PSPT archive. In order to discuss effects of both telescope operation and seeing on the results obtained, we analyzed a sample of higher resolution MLSO and Rome PSPT images available for year 2005.

Figure 9 shows the relative differences among contrast CLV computed from the higher quality images compared to those used for the whole study. On average, less resolved images yield a slight contrast reduction over the whole disk. This reduction increases toward the limb, up to about $1 \%$ at $\mu=0.2$. The small sample of features identified for the year 2005 is probably the main source of noise in these results. Notice that, due to the site location, MLSO images are delayed by more than ten hours relative to the Rome ones. Thus the direct comparison of features identified by the various methods on the images obtained at the two sites on the same day is limited by rotation and evolution effects.

These results indicate that a careful analysis of the temporal dependence of the image quality is essential for studying the possible variation with the cycle of the properties of the identified features. We find that photometric accuracy, spatial scale, and stray light-level of the Rome PSPT data vary along the whole period analyzed by an amount less than half the largest deviation of these quantities over each year of the sample. The image quality is thus uniform over this dataset.
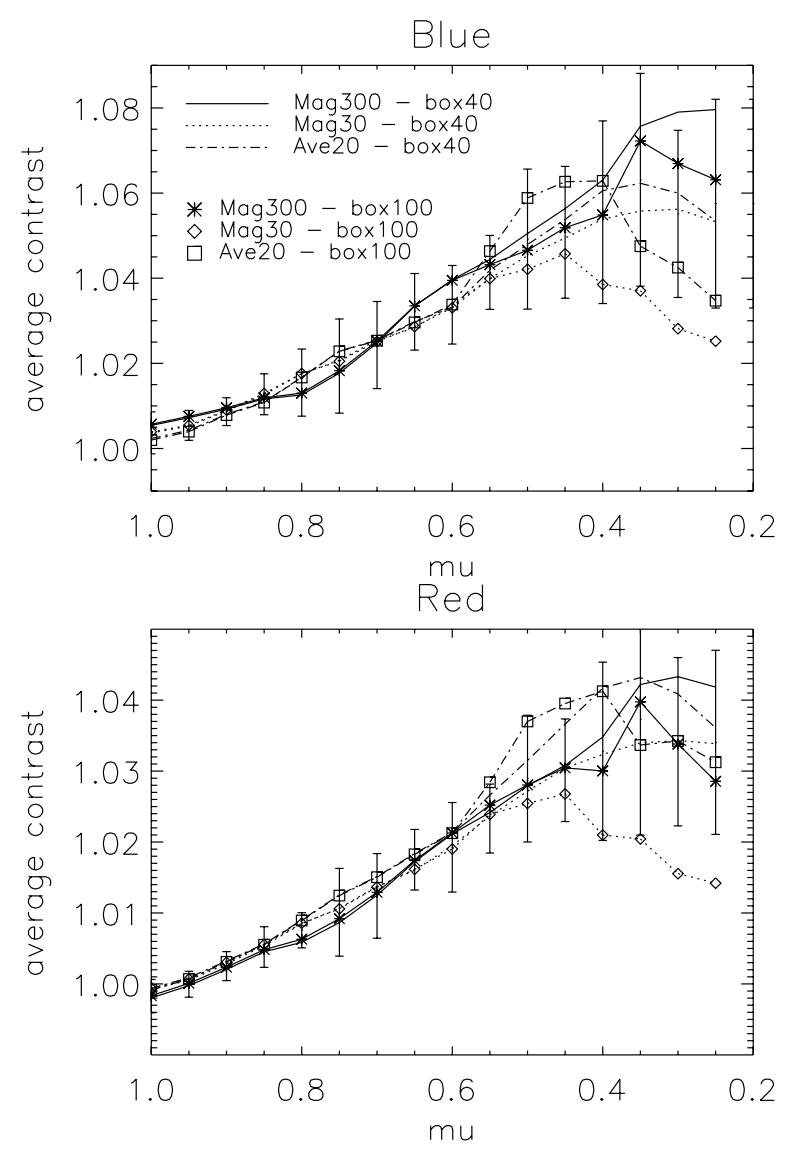

Fig. 10. Contrast CLV dependences obtained analyzing samples of magnetograms acquired with $30 \mathrm{~s}$ and $300 \mathrm{~s}$ integration times, as well as averages of 20 single $30 \mathrm{~s}$ magnetograms, by assuming two different processing parameters (running box side $=40$ and 100 pixels). For comparison, results presented in Sect. 4.1 are plotted with symbols and relative deviations.

The results presented were also obtained from analyzing MDI single magnetograms acquired with $30 \mathrm{~s}$ integration time. In order to discuss the effects of the data noise level on the results obtained, we analyzed two samples of MDI single magnetograms acquired in the year 2000, with $30 \mathrm{~s}$ and $300 \mathrm{~s}$ integration times (Mag30 and Mag300, hereafter). Moreover, we computed averages over 5 and 20 Mag30 single data (Ave 5 and Ave20, respectively), taken at a cadence of 1 per minute, centered at the time of the PSPT observation. Ortiz et al. (2006) found that the noise level of Mag300 data is 1.77 lower than that of the Mag30 magnetograms used for our study. On the other hand, the noise of Ave 5 and Ave20 data varies roughly as $\frac{1}{\sqrt{n}}$ of the Mag30 noise, where $n$ is the number of magnetograms averaged together. Notice that the different sets of magnetograms analyzed concern the same set of observational days, i.e. of solar features. All these magnetograms were pre-processed as described in Sect. 2.2, save for changes on the side length of the running box used.

Figure 10 shows that the contrast of features identified by the Mag300 data is higher than the one obtained from the analysis of Mag30 magnetograms, especially toward the limb. Nevertheless, the contrast CLV obtained with the two sets of data is rather similar. By contrast, notice the different CLV behavior obtained using the sample of Ave 20 data, as well as using the sets of same magnetograms processed with a wider running box. In particular, we applied a running box with a side length of 100 pixel, as used 
by Ortiz et al. (2002). We find that the contrast CLV obtained by these latter datasets shows a maximum at disk positions closer to the disk center, specifically around $\mu=0.4$. However, the difference between the results obtained by the various sets of magnetograms processed homogeneously, i.e. with the same running box, is smaller than the deviation of Mag30 contrast measurements presented in Sect. 4.1. For clarity, we show and discuss only Ave 20 results, since these are very similar to those obtained by the Ave 5 data.

These results show that our contrast measurements are slightly affected by a data noise-level reduction over almost the whole disk. This reduction can be achieved for example by analyzing MDI magnetograms acquired with $300 \mathrm{~s}$ integration time. By contrast, we show that the results obtained can be contaminated by the compensation applied to reduce the noise pattern of the data, such as the choice of the running box side length. We are aware that the noise level of Mag30 data is higher than that of the other magnetograms. However, the Mag30 data maximize the number of usable MDI data. Notice that the average and maximum values of the relative difference between the two noise patterns obtained with the 40 and 100 pixel boxes are $\approx 1.5 \%$ and $2 \%$, respectively, at disk positions $\mu>0.3$.

Finally, we find that the average contrast of the identified features at the disk center is positive, except for the red continuum measurements from Mag (Fig. 3). Indeed, this result is slightly sensitive to the operative definition of spots and pores and to the way the pixels belonging to these features are excluded from the identified facular regions. For instance, we find facular contrast values at disk center lower than those reported above by using a sunspot identification criterion based on feature size and intensity thresholding of umbra and penumbra regions separately. By this criterion, the deviations in measured values are larger. Notice that we have assumed the sunspot operative definition described in Sect. 3 to link our findings directly to those of some recent similar studies.

\subsection{Comparison with previous research}

\subsubsection{Contrast CLV}

The radiative properties of magnetic features in the solar atmosphere have been the object of numerous investigations. With respect to facular regions, these investigations have not given a clear picture. We show that the facular contrast CLV may also critically depend on the identification method used to discern features. In particular, the simultaneous use of five different identification methods applied to the same sample of data allows us to explain some of the differences existing among previously reported results.

Figure 11 shows the comparison of the results we obtained with those recently presented from similar studies (Lawrence et al. 1988; Ortiz et al. 2002; Walton et al. 2003; Foukal et al. 2004). We find that the average contrast of facular regions is essentially positive at the disk center in the PSPT blue and red bands. This result agrees with those previously obtained by authors who do not use identification methods based on magneticfield flux thresholding (Lawrence 1988; Lawrence Chapman \& Herzog 1988; Ahern \& Chapman 2000; Walton et al. 2003). In contrast, results obtained with these last methods (Foukal et al. 1990; Topka et al. 1992; Ortiz et al. 2002, 2006) show that facular regions at photospheric levels have negative contrasts at the disk center with respect to the neighboring quiet regions.

We show that, at the disk center, the identification method based on magnetic signal (Mag) seems to pick out slightly more

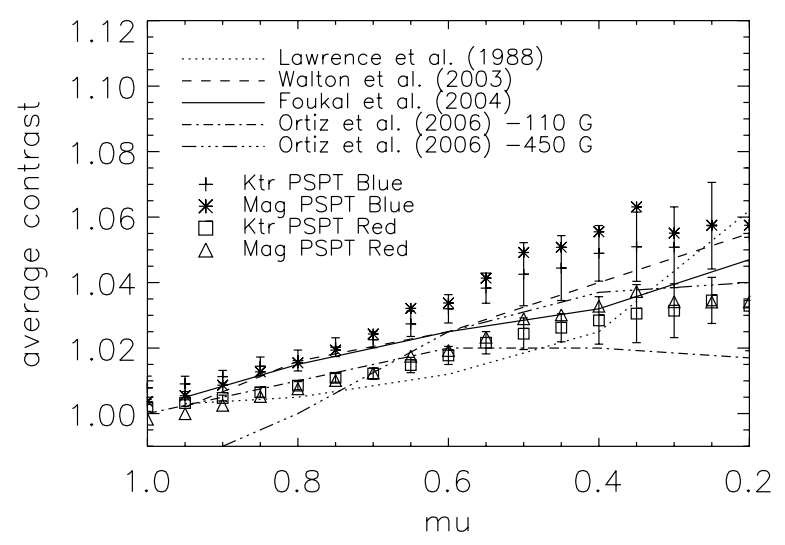

Fig. 11. Selected facular contrast CLV measurements plotted versus disk position. The symbols with error bars show the results obtained with the Ktr method, while those without error bars show the results by the Mag method. The different lines show the results of recent measurements of facular contrast presented in the literature.

negative $(<0.5 \%)$ features than those identified by the other methods based on intensity contrast $(K t r, N R, C H, B-R)$. Those are features that are not marked as spots on PSPT continuum observations but that have a lower contrast than the quiet sun. Nevertheless, the negative contrast values we measured with Mag largely differ from those presented in the literature, for example, by Ortiz et al. (2002) and Ortiz et al. (2006). In this comparison, the discrepancy in results obtained may depend on the different spectral sampling of PSPT continuum images with respect to MDI intensity ones.

Notice that, in agreement with our finding, Tritschler \& Uitenbroek (2006) have recently asserted by MHD simulations results that identification methods of features based on magnetic flux intensity give lower contrast measurements than methods based on intensity contrast. Unfortunately, a direct comparison of their results with those obtained by our study is not possible, because of the different spatial resolution ${ }^{5}$ and different spectral ranges considered.

We find a monotonic increase in the contrast as the heliocentric angle increases in all the methods we applied. This diskdependence agrees with the results obtained by Vögler (2005). From MHD simulations, this author shows that the bolometric contrast of facular features increases monotonically up to $\mu=0.2$ for features characterized by small magnetic signals and continues growing towards the limb for features with larger signals. This disk-dependence differs from the one presented by Ortiz et al. (2002) and Ortiz et al. (2006), which shows that features reach their maximum contrast at disk positions closer to the disk center. The decrease in the contrast reported by these authors toward the limb has been attributed to resolution effects by Domingo et al. (2005). Vögler (2005) explains the same decrease through differences in the spatial distribution of features smeared by the resolution element analyzed. Actually, we have shown that contrast values obtained with the Mag method applied to MDI full-disk magnetograms at disk positions $\mu<0.4$ may be contaminated by the compensation applied for the reduction in the data noise pattern.

\footnotetext{
5 About 100 times higher than that of the datasets analyzed for our study.
} 


\subsubsection{Wavelength, size, and time dependences}

The results obtained with all the methods show that relative contrasts in different continuum bands are roughly proportional to (wavelength) $)^{-1}$, as expected from a Planck distribution in the Wien approximation. Nevertheless, the curves drawn by our contrast measurements lie systematically above what is obtained with the blackbody approximation, for all the identification methods applied and for all the disk positions. Moreover, the distance between the measured curve and the one obtained with the approximation increases from the limb toward the disk center. These results confirm earlier findings by Chapman \& Mc Guire (1977) and Lawrence et al. (1988) from observations of smaller samples of facular features. Thus, significant errors may be introduced when monochromatic measurements of facular contrasts are used to estimate bolometric luminosity (Foukal et al. 2004) or irradiance excess.

We find that the maximum contrast of features increases with their size for all the three PSPT bands analyzed. On the other hand, the increase in the average contrast of chromospheric regions shows nearly a plateau for large features. This agrees with the results recently presented by Meunier (2003) and Walton et al. (2003). Notice that the flux tube model of facular features predicts this behavior for the average and maximum contrast from geometrical considerations of both the filling factor of flux elements and the viewpoint of the observer.

We find that the change in facular contrast CLV during the analyzed period is within $1 \%$. At chromospheric levels, Worden et al. (1998) find that intensity contrasts of bright features (in detail, plage, enhanced network, and active network) remain approximately constant over the solar cycle. At photospheric levels, Walton et al. (2003) and Ortiz et al. (2006) report that small features do not present a change in contrast with the cycle. Our result agrees with the one reported by Ortiz et al. (2006) who analyze MDI magnetograms and intensity images obtained from 1996 to 2001. The lower uncertainty associated to our result, compared to the one $(10 \%)$ reported by Ortiz et al., may be ascribed mostly to the lower photometric accuracy of MDI intensity measurements ${ }^{6}$ compared to the PSPT ones.

The steady contrast in time reported by this study supports the idea that facular flux tubes do not vary with solar cycle. Nevertheless, due to the spatial resolution of the data analyzed, we can only take those effects into account associated with the combined changes in both contrast and clustering of magnetic elements. Thus consistent high-resolution sub-arcsec observations performed at various activity levels are required to study this subject in greater depth.

Finally, the maximum-over-minimum ratio of the number of small-size features identified by the Ktr method agrees fully with the ratio (1.5) reported by Foukal et al. (1991) for network features. On the other hand, the ratio value estimated from the number of larger sized features is also in agreement with the one (3.5) reported by Meunier (2003) for magnetic features below $3 \times 10^{19} \mathrm{Mx}$.

\subsection{Comparison with semi-empirical models}

We now compare the results obtained with the outputs of some recent atmospheric model computations of facular features. In particular, we consider the three sets of one-dimensional semiempirical average models proposed by Fontenla et al. (2006),

6 The nominal noise level for MDI continuum 1-min measurement is $0.3 \%$.
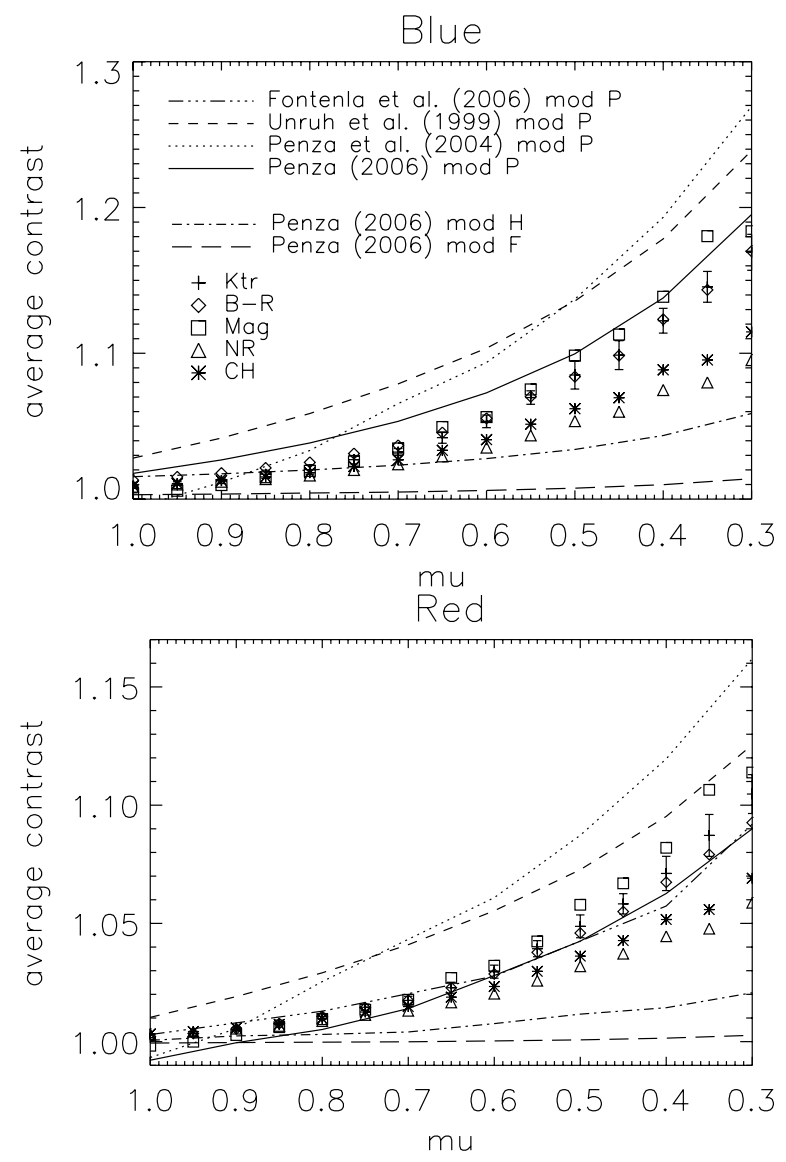

Fig. 12. Comparison of contrast CLV measurements with the output of semi-empirical atmospheric model computations ( $t o p=$ PSPT blue, bottom $=$ PSPT red measurements). The different lines show the results of model computations. Symbols represent the results obtained for the year 2000 by the different identification methods. For clarity, the deviation in measured values is plotted only for the results obtained with the $K t r$ method. Notice that Fontenla et al. (2006) model calculations are available only for the PSPT red band.

Unruh et al. (1999), Penza et al. (2004), and Penza (2006) ${ }^{7}$. These authors assumed complementary approaches, which differ in the data and approximation used.

The output of model computations for the PSPT measured bands were kindly provided by the authors of the models. Notice that, for this comparison, the measured contrast CLV has been corrected for a geometrical effect, as discussed by Penza et al. (2004). In brief, this correction considers the dilution effect on contrast measurements of features by the quiet sun at the increase of the heliocentric angle. This correction makes increasing modifications to the measured contrast CLV moving toward the limb. In particular, the contrast increase is about $3 \%$ and $1.8 \%$ at $\mu=0.5$ and about $15 \%$ and $9 \%$ at $\mu=0.2$ for the PSPT blue and red measurements, respectively.

Figure 12 shows the comparison of the CLV of limbcorrected contrasts with respect to those predicted by the models listed above. In particular, we considered the computed contrast of model $\mathrm{P}$, which is representative of facular regions in all the three sets of models. We find that the contrast CLV obtained by Ktr and Mag can be reproduced through a single atmospheric model with an offset ranging from $\approx 1$ to $5 \%$ over the whole disk

\footnotetext{
7 These models are obtained by applying the Penza et al. (2004) approach to the atmospheric stratifications proposed by Fontenla et al. (2006).
} 
up to $\mu=0.3$, depending on the model. The fraction of solar disk considered was reduced to $\mu \leq 0.3$, because this range is shared by all the computations. The relative differences among the computed and measured contrast values are 2-3 times larger than the relative deviation in the measurement results at the disk center, while they show very similar values at $\mu=0.3$. Moreover, we find that the contrast CLV measured with $\mathrm{NR}$ and $\mathrm{CH}$ is two and three times steeper than what is calculated (Penza 2006) assuming model $\mathrm{H}$, representative of plage regions on Fontenla et al. models, in the blue and red bands, respectively. The offset is $\approx 3 \%$ and $2 \%$ at $\mu=0.4$ for the PSPT blue and red bands, respectively. On the other hand, the agreement between measurements and computations (Penza 2006) of model F, representative of active network regions on Fontenla et al. models, is rather poor. The relative deviation results are $\approx 10 \%$ and $5 \%$ at $\mu=0.4$ for the PSPT blue and red bands measurements, independent of the identification method.

The agreement found between measured and modelled CLV dependences is quite important. Semi-empirical atmospheric models are in fact used for both static and dynamic numerical simulations of the solar atmosphere (see i.e. Okunev \& Kneer 2005; Steiner 2005) in order to reproduce high-resolution intensity and spectropolarimetric observations of facular regions.

\section{Conclusions}

We have presented a study of the contrast of facular features and its dependence on disk position, feature size and activity cycle. We also showed that the results obtained are quite sensitive to the identification method applied, as well as to the limited quality of the data analyzed. We then compared the results with recent finding of studies carried out with similar data, as well as with the output of most recent 1-D semi-empirical atmospheric models of facular regions.

These results were obtained analyzing moderate spatial resolution data, where these solar features appear as bright quite large patches with a mean size of a few kilo-arcsec. Although high-resolution observations have shown a rather small subarcsec granular appearance of these features, results obtained at moderate resolution are still quite important. For instance, static and dynamic numerical simulations currently used to obtain constraints to the physical description of these features (e.g. Steiner 2005; Vögler et al. 2005) make assumptions based on low-resolution results. In particular, the results of these simulations have to be smeared to get the quantitative comparisons with the observed features necessary for validating this approach. Moreover, at present, only results of moderate resolution observations allow us to study medium and large-scale activity features and to deepen the understanding of solar cycle variations. Finally, these observations allow us to use the results today of past synoptic programs. For instance, the results presented above can be used to study past total solar irradiance variations, such as Lockwood (2005) recently did, through results of old fulldisk observations and facular contrast measurements presented by Ortiz et al. (2002).

Acknowledgements. We are grateful to J. Fontenla and Y. Unruh for providing their model computations. The paper has been improved by comments from
J. Fontenla, M. Rast, and S. Solanki. I. E. thanks the "Solar Magnetism and Irradiance" team for helpful discussions. This team, chaired by V. Domingo, has been supported by the ISSI in Bern. We thank the referee for the detailed and constructive comments.

\section{References}

Ahern, S., \& Chapman, G. A. 2000, Sol. Phys., 191, 71

Allen's Astrophysical Quantities, The new edition 2000, ed. A. N. Cox (New York: Springer-Verlag, AIP Press)

Caccin, B., Ermolli, I., Fofi, M., \& Sambuco, A. M. 1998, Sol. Phys., 177, 295

Chapman, G. A., \& Sheeley, N. R. Jr. 1968, Sol. Phys., 5, 442

Chapman, G. A., \& Mc Guire, T. E. 1977, ApJ, 217, 657

Coulter, R. L., \& Kuhn, J. F. 1994, ASP Conf. Ser., 68, 37

Criscuoli, S., Rast, M. P., Ermolli, I., \& Centrone, M. 2007, A\&A, 461, 331

Domingo, V., Ortiz, A., Sanahuja, B., \& Cabello, I. 2005, Adv. Space Research, 35,345

Dunn, R. B., \& Zirker, J. B. 1973, Sol. Phys., 33, 281

Ermolli, I., Berrilli, F., \& Florio, A. 2003, A\&A, 412, 857

Fazzari, C., Ermolli, I., Centrone, M., Criscuoli, S., \& Giorgi, F. 2003, Mem. SAIt., 74, 667

Fontenla, J. M., Harder, J., Rottman, G., et al. 2004, ApJ, 605, L85

Fontenla, J. M., Avrett, E., Thuillier, G., \& Harder, J. 2006, ApJ, 639, 441

Foukal, P., \& Duvall, T. Jr. 1985, ApJ, 296, 739

Foukal, P., Little, R., Graves, J., Rabin, D., \& Lynch, D. 1990, ApJ, 353, 712

Foukal, P., Harvey, K., \& Hill, F. 1991, ApJ, 383, L89

Foukal, P., Bernasconi, P., Eaton, H., \& Rust, D. 2004, ApJ, 611, L57

Frazier, E. N. 1971, Sol. Phys., 21, 42

Harvey, K. L., \& White, O. R. 1999, ApJ, 515, 812

Keller, C. U., Schüssler, M., Vögler, A., \& Zakharov, V. 2004, ApJ, 607, L59

Krivova, N. A., Solanki, S. K., Fligge, M., \& Unruh, Y. C. 2003, A\&A, 399, L1 Lawrence, J. K. 1988, Sol. Phys., 116, 17

Lawrence, J. K., Chapman, G. A., \& Herzog, A. D. 1988, ApJ, 324, 1184

Lites, B. W., Scharmer, G. B., Berger, T. E., \& Title, A. M. 2004, Sol. Phys., 221, 65

Lockwood, M. 2005, in The Sun, Solar Analogs and the Climate (Springer), 109 Mehltretter, J. P. 1974, Sol. Phys., 38, 43

Meisner, R. W., \& Rast, M. P. 2002, American Astronomical Society, 200th AAS Meeting, 55.13, BAAS, 34, 734

Meunier, N. 2003, A\&A, 405, 1107

Nesme-Ribes, E., Meunier, N., \& Collin, B. 1996, A\&A, 308,2213

Ortiz, A., Solanki, S. K., Domingo, V., Fligge, M., \& Sanahuja, B. 2002, A\&A, 388,1036

Ortiz, A., Domingo, V., \& Sanahuja, B. 2006, A\&A, 452, 311

Okunev, O. V., \& Kneer, F. 2005, A\&A, 439, 323

Penza, V. 2006, private communication

Penza, V., Caccin, B., Ermolli, I., Centrone, M., \& Gomez, M. T. 2003, ESA SP535, 299

Penza, V., Caccin, B., Ermolli, I., \& Centrone, M. 2004, A\&A, 413, 1115

Richardson, R. S. 1933, ApJ, 78, 359

Scherrer, P. H., Bogart, R. S., Bush, R. I., et al. 1995, Sol. Phys., 162, 129

Solanki, S. K. 1993, Space Sci. Rev., 63, 1

Solanki, S. K. 1994 (Cambridge: Cambridge University Press), IAU Coll., 143, 226

Solanki, S. K., \& Krivova, N. A. 2004, Sol. Phys., 224, 197

Spruit, H. C. 1977, Thesis University of Utrecht, The Nederlands Steiner, O. 2005, A\&A, 430, 691

Topka, K. P., Tarbell, T. D., \& Title, A. M. 1992, ApJ, 396, 351

Tritschler, A., \& Uitenbroek, H. 2006, ApJ, 648, 741

Turmon, M., Pap, J. M., \& Mukhtar, S. 2002, ApJ, 568, 396

Unruh, Y. C., Solanki, S. K., \& Fligge, M. 1999, A\&A, 345, 635

Vögler, A. 2005, Mem. SAIt. 76, 842

Vögler, A., Shelyag, S., Schüssler, M., et al. 2005, A\&A, 429, 335

Walton, S. R., Preminger, D., \& Chapman, G. A. 2003, Sol. Phys., 213, 301

Wenzler, T., Solanki, S. K., Krivova, N. A., \& Fluri, D. M. 2004, A\&A, 427, 1031

Wenzler, T., Solanki, S. K., \& Krivova, N. A. 2005, A\&A, 432, 1057

Worden, J. R., White, O. R., \& Woods, T. N. 1998, ApJ, 496, 998 